\section{ORIGINAL RESEARCH}

E.G. Kang

S.J. Jeon

S.S. Choi

C.J. Song

I.K. Yu

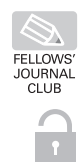

\title{
Diffusion MR Imaging of Hypoglycemic Encephalopathy
}

BACKGROUND AND PURPOSE: MR imaging features of HE have not been fully established. The purpose of this study was to determine the topographic distribution and DWI findings of $\mathrm{HE}$.

MATERIALS AND METHODS: We retrospectively evaluated HE MR imaging $(n=11)$. The topographic distribution of the lesions was evaluated on routine MR imaging, and DWI SI and ADC values were assessed. The ADC value of involved lesions was compared with the noninvolved subcortical WM area by use of the paired $t$ test.

RESULTS: MR images demonstrated bilateral diffusion-restrictive lesions in the posterior limb of the IC $(n=6)$, cerebral cortex $(n=8), \mathrm{CR}(n=7), \mathrm{CS}(n=9)$, hippocampus $(n=4)$, and BG $(n=1)$. The mean $A D C$ value of lesions was $448.82 \pm 92.34 \times 10^{-6} \mathrm{~mm}^{2} / \mathrm{s}$ compared with the mean ADC value of noninvolved lesions $\left(837.72 \pm 62.14 \times 10^{-6} \mathrm{~mm}^{2} / \mathrm{s}\right)$; this difference was statistically significant $(P<$ .000). The lesions showed complete resolution on follow-up DWI for 6 patients. Three patients with cortical involvement of $\geq 2$ lobes showed partial recovery or death, but most of the other patients with WM involvement or cortical involvement in only 1 lobe experienced complete recovery.

CONCLUSIONS: The topographic localization of the lesions was the posterior limb of the IC, cerebra cortex, CR, CS, hippocampus, and BG. Most HE lesions probably correspond to areas of reversible cytotoxic edema as seen on DWI, which can predict the prognosis of HE according to the degree of lesion extent.

\begin{abstract}
ABBREVIATIONS: $A D C=$ apparent diffusion coefficient; $A P N=$ acute pyelonephritis; $B G=$ basal ganglia; $\mathrm{BST}=$ blood sugar test; $\mathrm{CC}=$ corpus callosum; $\mathrm{CR}=$ corona radiata; $\mathrm{CS}=$ centrum semiovale; $\mathrm{DM}=$ diabetes mellitus; $\mathrm{DWI}=$ diffusion-weighted image; FLAIR = fluid-attenuated inversion recovery; $\mathrm{FL}=$ frontal lobe; $\mathrm{HE}=$ hypoglycemic encephalopathy; $\mathrm{IC}=$ internal capsule; $\mathrm{IN}=$ insular lobe; $\mathrm{MCA}=$ middle cerebral artery; $\mathrm{MRI}=\mathrm{MR}$ imaging; $\mathrm{NSA}=$ number of signals averaged; $\mathrm{OC}=$ occipital lobe; $\mathrm{PA}=$ parietal lobe; $\mathrm{SI}=$ signal intensity; $\mathrm{TL}=$ temporal lobe; $\mathrm{WM}=$ white matter
\end{abstract}

H ypoglycemia is the sudden decrease in serum glucose level $<50 \mathrm{mg} / \mathrm{dL}$, and the organ systems that manifest the signs and symptoms are the central and autonomic nervous system. Diverse neurologic manifestations of hypoglycemia have been reported frequently. These neurologic symptoms range from focal neurologic deficits to permanent dysfunction or death. ${ }^{1-3}$ Neuropathologic studies have demonstrated that the cerebral cortex, hippocampus, and BG are commonly affected sites in severe hypoglycemia; however, the cerebellum and brain stem are usually spared. ${ }^{4-10}$ In addition, unlike hypoxic damage, the occipital cortex, dorsofrontal cortex, and hippocampus may be more resistant to prolonged hypoglycemia. ${ }^{9}$ Some studies have described MR imaging of hypoglycemic brain injury, though these studies have usually been confined to case reports; in most of the reported studies, DWI is useful in evaluating severe HE. Reversible diffusion restriction has been found in most cases. ${ }^{1,5-7,10-16}$ However, most

\section{Received June 23, 2009; accepted after revision July 28}

From the Department of Radiology (E.G.K., S.J.J., S.S.C.), Wonkwang University Hospital, Chunbuk, Republic of Korea; Department of Radiology (C.J.S.), Chungnam National University Hospital, Deajeon, Republic of Korea; and Department of Radiology (I.K.Y.), Eulji University Hospital, Deajeon, Republic of Korea.

Please address correspondence to Se Jeong Jeon, MD, Department of Radiology, Wonkwang University Hospital, 344-2, Shinyong-dong, Iksan, Chunbuk, 570-711, Republic of Korea; e-mail: medicalq@hanmail.net

\section{Indicates open access to non-subscribers at www.ajnr.org}

DOI 10.3174/ajnr.A1856 studies have been case reports, and MR imaging features of hypoglycemic brain injury have not been fully established.

The purpose of this study was to determine the topographic distribution and DWI findings of HE.

\section{Materials and Methods}

\section{Patients}

From March 2006 to February 2009, we retrospectively studied 14 patients with hypoglycemic coma in whom brain MR imaging was performed. Patients were admitted to the emergency department of 3 institutions (Wonkwang University Hospital, Chungnam National University Hospital, and Eulji University Hospital). We excluded 3 patients because 2 of the 3 did not have ADC map images and 1 patient was confirmed as having encephalitis, not HE. Therefore, 11 patients were included in this study. The study population consisted of 7 women and 4 men, with a mean age of 55.5 years (age range, 43-76 years). We reviewed medical records to assess clinical parameters, such as the initial blood sugar level, predisposing factors, neurologic symptoms and signs, and outcome of recovery after glucose injection.

\section{MR Imaging}

All 11 patients underwent MR imaging within 72 hours after symptom onset. DWI was performed in all patients, and 6 patients underwent follow-up MR imaging. MR imaging was performed with the use of a 1.5T MR imaging system (Gyroscan Intera; Philips Medical Systems, Best, the Netherlands). Imaging sequences of the brain included 


\begin{tabular}{|c|c|c|c|c|c|c|c|c|c|c|c|c|}
\hline \multirow{2}{*}{$\begin{array}{l}\text { Patient } \\
\text { No. }\end{array}$} & \multicolumn{4}{|c|}{ WM } & \multicolumn{6}{|c|}{ Gray matter } & \multirow[b]{2}{*}{ Hippocampus } & \multirow[b]{2}{*}{ Bilaterality } \\
\hline & $\mathrm{CS}$ & $\mathrm{CR}$ & IC & $\mathrm{CC}$ & $\mathrm{FL}$ & $\mathrm{PA}$ & $\mathrm{TL}$ & OC & IN & $\mathrm{BG}$ & & \\
\hline 1 & + & + & + & - & - & + & - & - & - & - & - & + \\
\hline 2 & + & + & - & - & + & - & - & - & - & - & - & + \\
\hline 3 & - & - & - & - & + & + & - & + & - & - & + & + \\
\hline 4 & + & + & + & - & + & + & - & - & - & - & - & + \\
\hline 5 & + & + & + & - & + & - & - & - & - & - & - & + \\
\hline 6 & + & + & + & - & - & - & - & - & - & - & + & $-b$ \\
\hline 7 & + & - & + & - & + & - & - & - & - & - & - & + \\
\hline 8 & + & - & - & - & + & - & - & - & - & - & - & + \\
\hline 9 & + & + & + & - & - & - & - & - & - & - & - & + \\
\hline 10 & + & + & - & - & - & - & - & - & - & - & + & + \\
\hline 11 & - & - & - & - & + & + & + & + & + & + & + & + \\
\hline
\end{tabular}

a + indicates the presence of a lesion at each anatomic location; -, no abnormality at each anatomic location on MR image.

b Unilateral involvement because there was an old infarction at the right MCA territory.

spin-echo T1-weighted images, T2-weighted images, FLAIR images, enhanced T1-weighted images with gadopentetate dimeglumines, and DWI. From DWI, ADC maps were calculated. The MR imaging parameters were as follows: $400-50 \mathrm{~ms} / 10-12 \mathrm{~ms} / 2 / 190-192 \times 320-$ 512 (TR/TE/NSA/matrix) for spin-echo T1-weighted images; 4000$4851 \mathrm{~ms} / 120 \mathrm{~ms} / 1-2 / 15 / 240-256 \times 320-512$ (TR/TE/NSA/ echo-train/matrix) for turbo spin-echo T2-weighted images; and 8000-11,000 ms/127-140 ms/2800 ms/1/18/190-192 × 320-512 (TR/TE/TI/NSA/echo-train/matrix) for turbo FLAIR images. The other parameters were the following: section thickness, $5 \mathrm{~mm}$ with a 1.5- to 1.8 -mm gap; FOV, $220-286 \times 20-220 \mathrm{~mm}$. DWI was performed with single-shot spin-echo with 2 diffusion-sensitivity values of 0 and $1000 \mathrm{~s} / \mathrm{mm}^{2}$ along all 3 axes.

\section{Imaging Analysis}

Two radiologists (S.J.J. and S.S.C) retrospectively evaluated the MR images in consensus. SIs of lesions were visually assessed on DWI and ADC maps, and the location of lesions was determined on the basis of their relatively high SI on DWI and low SI on ADC. The anatomic locations of lesions and the existence of enhancement on contrastenhanced T1-weighted images were assessed. To quantify the ADC value of lesions, we manually drew regions of interest along high-SI lesion boundaries on DWI, and regions of interest were copied to the same location on ADC maps. We also measured the ADC values of normal WM in the noninvolved subcortical WM. In 6 patients with follow-up MR imaging, interval changes of lesions were assessed.

\section{Statistical Analysis}

Statistical analysis was performed with commercially available software (Version 12.0; SPSS, Chicago, Illinois). The mean ADC values of involved areas were compared with those of uninvolved WM by use of the paired $t$ test. A $P$ value $<.05$ was considered to indicate a statistically significant difference.

\section{Results}

The mean initial blood sugar level was $19.6 \mathrm{mg} / \mathrm{dL}$ (range, 3-36 mg/dL) at the emergency department. Eight of the 11 patients had DM. Predisposing factors of hypoglycemia included inflammatory disease $(n=3)$, pelvic fracture with sepsis $(n=1)$, malnutrition $(n=1)$, iatrogenic DM medication $(n=1)$, iatrogenic insulin injection $(n=1)$, renal stone with hydronephrosis $(n=1)$, and unknown $(n=3)$. The initial neurologic symptoms of all patients included a change of mental status (confusion to coma). No patients experienced seizures before the initial MR imaging study. Arterial blood pressure of all patients was normal, and there was no hypoxia. Most of the patients had an improved mental status after the serum blood sugar level was normalized, but 2 patients died.

All 11 patients underwent MR imaging within a few hours to 3 days of the time of hospital admission. Table 1 shows the distribution of lesions. In most patients, the lesions were bilateral except for 1 patient. This patient (patient 6) had postinfarcted encephalomalacia located at the right MCA territory. Distribution of the lesions was as follows: the IC $(n=6,54 \%)$, hippocampus $(n=4,36 \%)$, cortex $(n=8,73 \%)$, CR $(n=7$, $64 \%)$, and CS $(n=9,82 \%)$. Only 1 patient had a lesion that involved the BG. Seven patients (64\%) showed involvement of the CS and CR (Fig 1). Involvement of the cortex was bilateral and did not match typical arterial territories. In 6 patients, there was patchy noncontiguous involvement at the frontal or parietal lobe. Two patients had extensive confluent cortical and hippocampal involvement without deep WM involvement (Fig 2). None of the lesions were enhanced on contrastenhanced T1-weighted images.

Lesions were visible on FLAIR images and on DWI and were more prominent on DWI in 9 patients. Table 2 shows the ADC values on initial MR imaging in all patients. The DWI SI of lesions was high, and ADC values were decreased. The mean ADC values were $448 \times 10^{-6} \mathrm{~mm}^{2} / \mathrm{s}$ (range, $248-578 \times 10^{-6}$ $\left.\mathrm{mm}^{2} / \mathrm{s}\right)$ in affected areas and $837 \times 10^{-6} \mathrm{~mm}^{2} / \mathrm{s}$ in the normal WM (range, 759-946 $\times 10^{-6} \mathrm{~mm}^{2} / \mathrm{s}$ ). Moreover, the difference in the ADC values was statistically significant $(P<.000)$. Six patients underwent follow-up MR imaging within 19 days (range, 4-19 days) of the time of initial MR imaging, and images were accessed visually and compared with initial DWI with ADC maps. Diffusion-restrictive lesions on initial MR imaging had completely disappeared on follow-up MR imaging in 6 patients (Fig 3).

Seven patients were discharged within 3 weeks of admission without significant neurologic deficits, and 1 patient showed partial recovery. However, 2 patients with extensive cortical involvement died (patients 3 and 11). One patient showed no change in mental status, and the patient was transferred to another hospital.

\section{Discussion}

In this study, we described brain MR diffusion imaging features in 11 patients who had severe hypoglycemia due to 

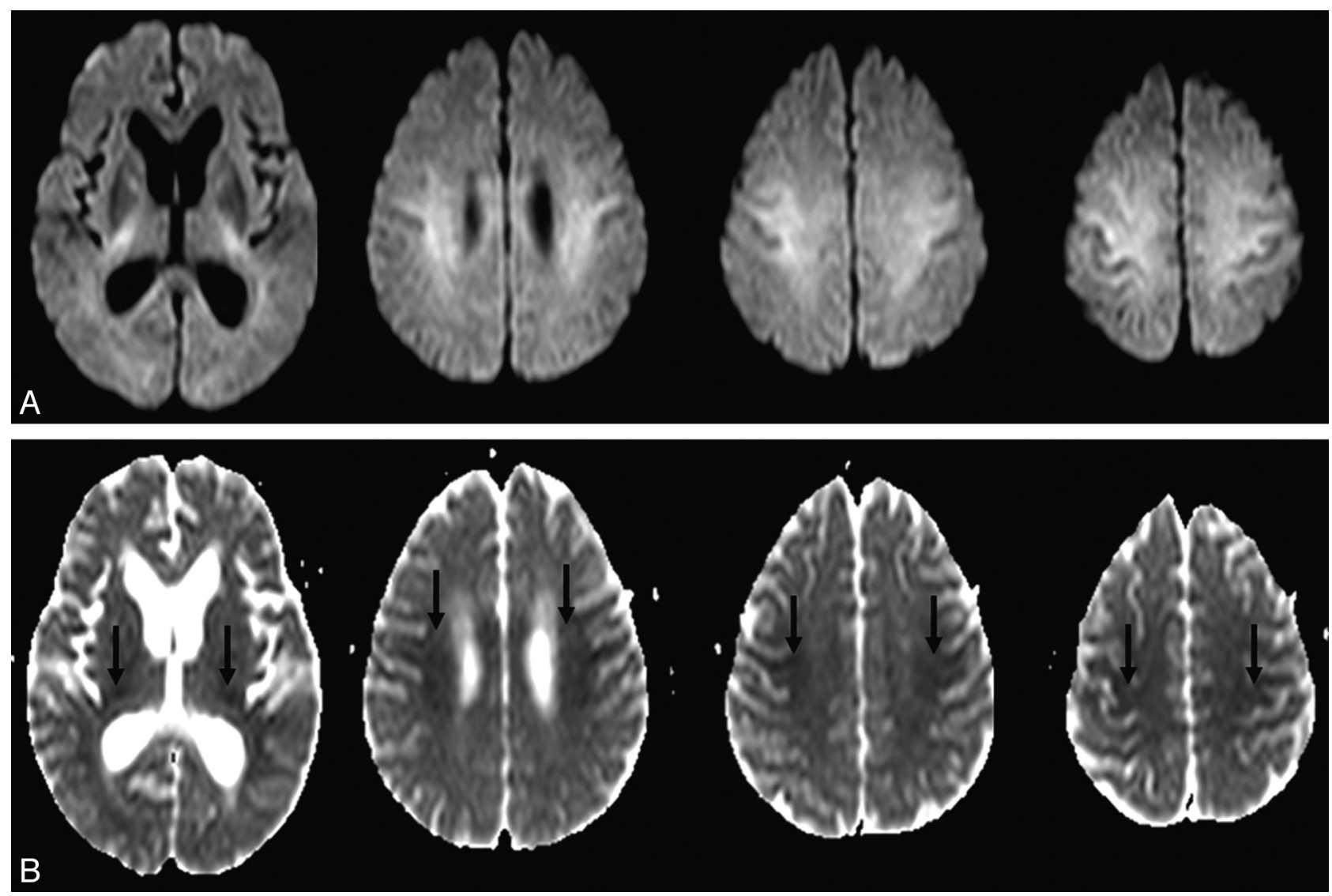

Fig 1. A 63-year-old woman (patient 4) was found unconscious. DWI on admission shows hyperintense lesions in the IC, CR, and CS (A) with reduced $A D C$ values (arrows, $B)$. These lesions are bilateral and symmetric.

various causes. To the best of our knowledge, this is the first study of a large case series with HE.

Hypoglycemia can be induced by overuse of insulin or oral hypoglycemic agents, undiagnosed insulinoma, or other medical diseases such as sepsis or renal or hepatic failure. ${ }^{15} \mathrm{Hypo-}$ glycemia can cause various neurologic symptoms including profound memory loss, transient motor deficits, a persistent vegetative state, and death in $2 \%-4 \%$ of cases. ${ }^{1-3,13}$

Brain MR imaging is a useful technique to evaluate severe HE. Among other sequences, DWI is a special technique that can measure the alteration of the diffusion of water within the extracellular space and between intracellular and extracellular spaces. ${ }^{17}$ Some authors have reported DWI and ADC findings of HE. ${ }^{1-3,5-16,18}$ According to previous reports, the lesions show reversible cytotoxic edema at the cerebral cortex, ${ }^{14,15,18}$ hippocampus, ${ }^{19}$ splenium, ${ }^{7,11,12,16}$ IC, ${ }^{13,16}$ and cerebral WM. ${ }^{5,8}$ These diffusion alterations are encountered not only in acute ischemia but also in hypoglycemia itself, and in vivo animal model studies have demonstrated diffusion alterations due to hypoglycemia. ${ }^{10,11,20}$

The pathomechanism of HE still remains unclear. Some pathogenetic mechanisms for diffusion restriction in HE that have been proposed include the following: 1) energy failure, 2) excitotoxic edema, and 3) asymmetric cerebral blood flow. First, severe hypoglycemia induces neurochemical changes. Glucose deprivation leads to arrest of protein synthesis in many regions, incomplete energy failure and loss of ion homeostasis, cellular calcium influx, and intracellular alkalosis.
Consequently, neuroactive amino acid (aspartate) release into the extracellular space occurs and results in selective neuronal necrosis, predominantly in the cerebral cortex, caudoputamen, and hippocampus. ${ }^{10,11,13,21}$ However, protein synthesis in the cerebellum, brain stem, and hypothalamus remains unaffected because of the greater activity of the glucose transport mechanisms. ${ }^{21-23}$

Second, excitotoxic edema is a cytotoxic form due to increased extracellular glutamate. The presence of glutamate leads to calcium and sodium entry into the cell and induces apoptosis. In contrast to cytotoxic edema, excitotoxic edema does not imply neuronal damage, because glutamate induces edema of glial cells and myelinic sheaths might protect axons from intracellular edema and irreversible damage. In addition, glutamate reuptake systems are not impaired in hypoglycemia. According to these mechanisms, HE is usually transitory and DWI abnormalities normalize with time or following removal of the causative pathologic factors. ${ }^{12,24}$

Last, when hypoperfusion complicates hypoglycemia, the brain is not exposed to an equal fall in perfusion. Due to the focal loss of autoregulation, the frontal and parietal lobe areas have grossly decreased cerebral flow, whereas the cerebellum and brain stem show almost no fall in local cerebral blood flow. ${ }^{23,25-27}$ MR imaging in our patients showed involvement of the same areas. Most of our patients had involvement of the cortex, and all cortical lesions were located in the frontal and parietal lobes. The MR imaging findings of our patients were in accord with the proposed mechanisms. 

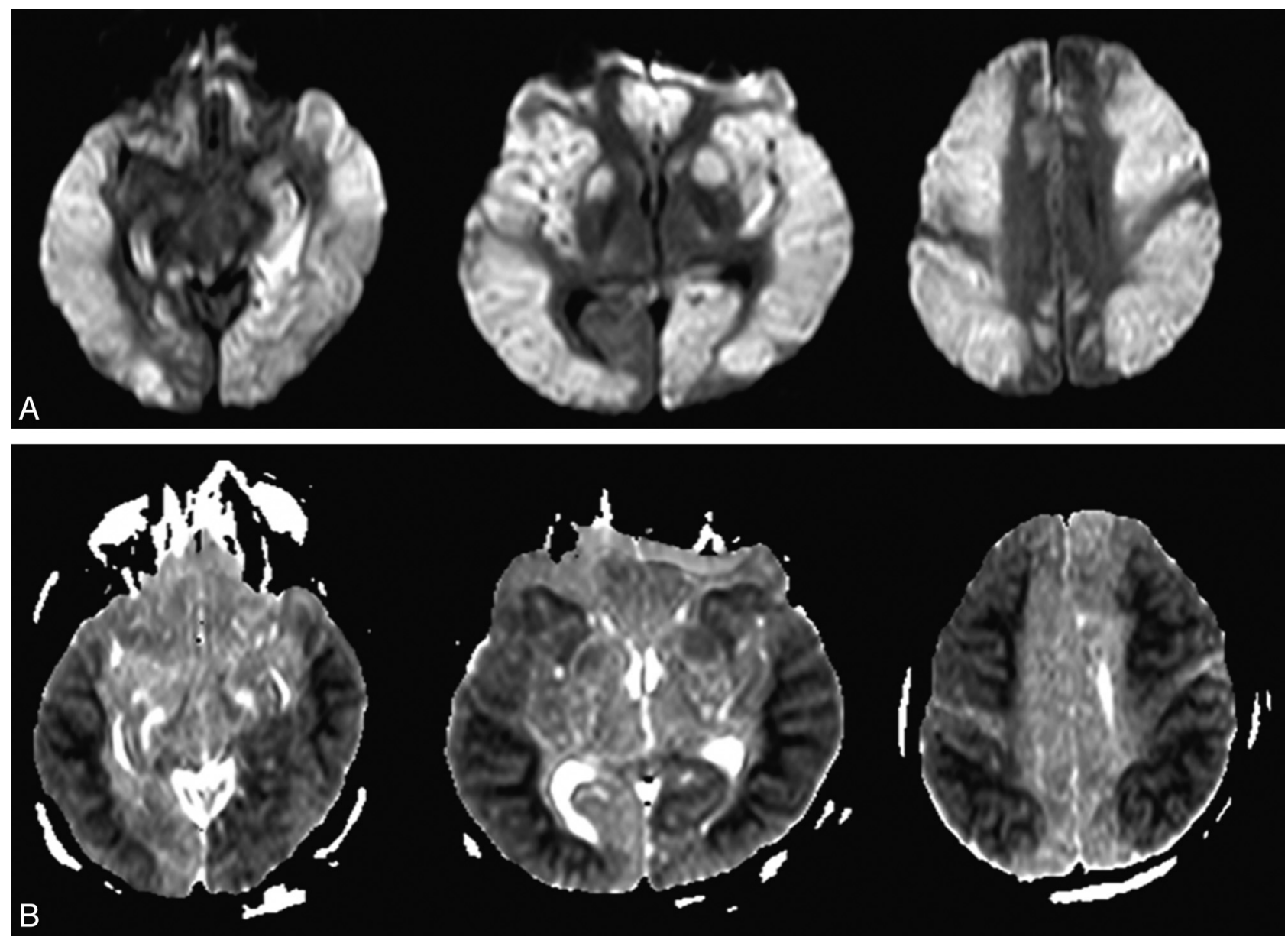

Fig 2. A 45-year-old man (patient 11) was found in a coma. A, DWI shows bilaterally asymmetric confluent hyperintense lesions in the frontal, parietal, insular, temporal, occipital cortices, and $\mathrm{BG}$. $B$, On ADC maps, these lesions show low SI, but the WM was spared.

\begin{tabular}{|c|c|c|c|c|c|}
\hline \multirow[b]{2}{*}{$\begin{array}{l}\text { Patient } \\
\text { No. }\end{array}$} & \multicolumn{3}{|c|}{ MR Imaging Findings } & \multirow[b]{2}{*}{$\begin{array}{l}\text { Follow-Up } \\
\text { MR Imaging }\end{array}$} & \multirow[b]{2}{*}{$\begin{array}{l}\text { Interval between } \\
\text { Initial and Follow-U } \\
\text { (Day) }\end{array}$} \\
\hline & SI on DWI & $\begin{array}{l}\text { ADC Value of Lesion } \\
\left(10^{-6} \mathrm{~mm}^{2} / \mathrm{s}\right)\end{array}$ & $\begin{array}{c}\text { ADC of the } \\
\text { Non-Involved Area } \\
\left(10^{-6} \mathrm{~mm}^{2} / \mathrm{s}\right)\end{array}$ & & \\
\hline 1 & High & $542 \pm 31.37$ & $946 \pm 52.50$ & Reversible & 11 \\
\hline 2 & High & $416 \pm 33.69$ & $837 \pm 117.90$ & No & \\
\hline 3 & High & $432 \pm 55.79$ & $794 \pm 52.50$ & No & \\
\hline 4 & High & $578 \pm 40.93$ & $914 \pm 45.04$ & No & \\
\hline 5 & High & $496 \pm 33.31$ & $784 \pm 43.93$ & Reversible & 18 \\
\hline 6 & High & $413 \pm 26.15$ & $882 \pm 95.70$ & Reversible & 19 \\
\hline 7 & High & $458 \pm 35.21$ & $786 \pm 61.04$ & Reversible & 6 \\
\hline 8 & High & $486 \pm 28.62$ & $892 \pm 50.33$ & Reversible & 4 \\
\hline 9 & High & $514 \pm 32.54$ & $788 \pm 81.04$ & Reversible & 8 \\
\hline 10 & High & $354 \pm 42.32$ & $759 \pm 43.42$ & No & \\
\hline 11 & High & $248 \pm 70.13$ & $833 \pm 84.22$ & No & \\
\hline
\end{tabular}

${ }^{a} \mathrm{ADC}$ values are presented as the mean $\pm \mathrm{SD}$.

The prognosis or neurologic sequelae of HE depends on the severity and duration of hypoglycemia. ${ }^{2}$ Some studies have reported that DWI may be useful to predict outcome. ${ }^{3,6,16,17}$ If hyperintensity lesions are detected in WM such as the CC, IC, or CR and the hyperintensity lesions regress on follow-up images, the patient will likely recover without a neurologic deficit. ${ }^{5,7,16}$ However, if lesions are detected in the cerebral cortex,
BG, or hippocampus and the lesions do not regress on second imaging, the outcome will be poor. ${ }^{2,5,9,18,28}$ In our study, prognosis of patients was similar, as reported previously. However, there were some differences. First, most of our patients who had frontal or parietal-cortical involvement did not have fatal consequences, only patients with diffuse and extensive cortical involvement. Second, the hippocampus was involved in $4 \mathrm{pa}-$ 

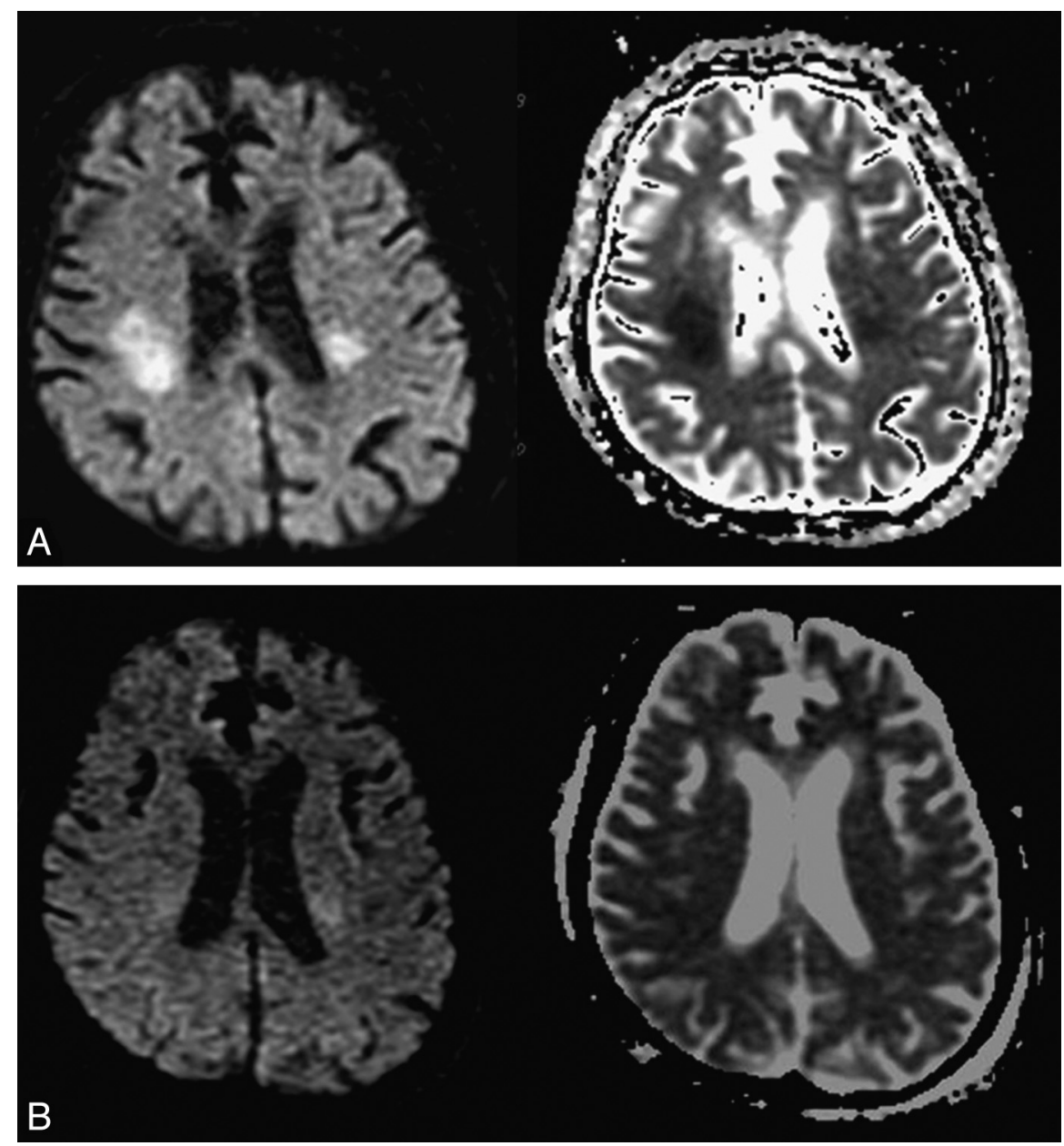

Fig 3. A 75-year-old woman (patient 7) was found in a confused mental state. $A$, Initial DWI on admission shows hyperintense lesions in the IC and CR, with reduced $A D C$ values. $B$, Follow-up DWI obtained 4 days after symptom improvement shows that the hyperintense lesions have disappeared, with normalization of ADC values.

tients (patients 3, 6, 10, and 11). Two of the 4 patients (patients 3 and 11) with diffuse cortical involvement died, but the other patients (patients 6 and 10) without cortical involvement had complete recovery. Therefore, in our opinion, hippocampal involvement does not predict a poor outcome with hypoglycemia. Furthermore, frontal and parietal cortical involvement was correlated with a worse outcome than only WM involvement but with a better outcome than diffuse cortical involvement or BG involvement.

Several different conditions, including seizure, drug toxicity, viral encephalitis, and metabolic encephalopathy, have reversible diffusion restrictions similar to those in hypoglycemia. ${ }^{14}$ However, most of these conditions are associated with other specific abnormalities. Therefore, a diagnosis of HE is not difficult to distinguish from several other conditions, even if the conditions show reversible diffusion abnormalities on MR imaging.

Our study has some limitations. First, it was retrospective, and the possibility of selection bias exists. Second, the number of patients was low, and follow-up MR imaging was performed for only a limited number of patients. Third, most patients were elderly and had other underlying medical illnesses in addition to hypoglycemia. Therefore, an underlying medical illness may not only have been a predisposing factor for hypoglycemia but could also have affected the clinical outcome of patients.

\section{Conclusions}

MR imaging findings in HE include reversible diffusion restriction at characteristic locations. These findings can be helpful in the differential diagnosis distinguishing HE from other neurologic conditions that have diffusion restrictions, such as stroke, epilepsy, and metabolic encephalopathy. In addition, DWI can predict the prognosis of patients with HE.

\section{References}

1. Bottcher J, Kunze A, Kurrat C, et al. Localized reversible reduction of apparent diffusion coefficient in transient hypoglycemia-induced hemiparesis. Stroke 2005;36:e20-22

2. Finelli PF. Diffusion-weighted MR in hypoglycemia coma. Neurology 2001;57:933-35

3. Shirayama H, Ohshiro Y, Kinjo Y, et al. Acute brain injury in hypoglycemiainduced hemiplegia. Diabet Med 2004;21:623-24

4. Auer RN, Wieloch T, Olsson Y, et al. The distribution of hypoglycemic brain damage. Acta Neuropathol 1984;64:177-91

5. Aoki T, Sato T, Hasegawa K, et al. Reversible hyperintensity lesion on diffusion-weighted MRI in hypoglycemic coma. Neurology 2004;27:392-93

6. Chan R, Erbay S, Oljeski S, et al. Case report: hypoglycemia and diffusionweighted imaging. J Comput Assist Tomogr 2003;27:420-23

7. Marya J, Endoh H, Watanabe H, et al. Rapid improvement of diffusion- 
weighted imaging abnormalities after glucose infusion in hypoglycemic coma. J Neurol Neurosurg Psychiatry 2007;78:102-03

8. Mori F, Nishie M, Houzen H, et al. Hypoglycemic encephalopathy with extensive lesions in the cerebral white matter. Neuropathology 2006;26:147-52

9. Cho SJ, Minn YK, Kwon KH. Severe hypoglycemia and vulnerability of the brain. Arch Neurol 2006;63:138

10. Hasegawa Y, Formato JE, Latour LL, et al. Severe transient hypoglycemia causes reversible change in the apparent diffusion coefficient of water. Stroke 1996;27:1648-56

11. Kim JH, Roh JH, Koh SB. Reversible injury of internal capsule and splenium in a patient with transient hypoglycemic hemiparesis. Cerebrovasc Dis 2006;22:282-83

12. Gallucci M, Limbucci N, Paonessa A, et al. Reversible focal splenial lesions. Neuroradiology 2007;49:541-44

13. Albayram S, Ozer H, Gokdemir S, et al. Reversible reduction of apparent diffusion coefficient values in bilateral internal capsules in transient hypoglycemia-induced hemiparesis. AJNR Am J Neuroradiol 2006;27:1760-62

14. Maekawa S, Aibiki M, Kikuchi K, et al. Time-related changes in reversible MRI findings after prolonged hypoglycemia. Clin Neurol Neurosurg 2006;108: 511-13

15. Lo L, Tan AC, Umapathi T, et al. Diffusion-weighted MR imaging in early diagnosis and prognosis of hypoglycemia. AJNR Am J Neuroradiol 2006;27:1222-24

16. Terakawa $\mathrm{Y}$, Tsuyuguchi N, Nunomura K, et al. Reversible diffusion-weighted imaging changes in the splenium of the corpus callosum and internal capsule associated with hypoglycemia: case report. Neurol Med Chir (Tokyo) 2007;47:486-88

17. Le Bihan D. The "wet mind": water and functional neuroimaging. Phys Med Biol 2007;52:R57-90
18. Yanagawa Y, Isoi N, Tokumaru AM, et al. Diffusion-weighted MRI predicts prognosis in severe hypoglycemic encephalopathy. J Clin Neurosci 2006;13:696-99

19. Boeve BF, Bell DG, Noseworthy JH. Bilateral temporal lobe MRI changes in uncomplicated hypoglycemic coma. Can J Neurol Sci 1995;22:56-58

20. De Crespigny AJ, Röther J, Beaulieu C, et al. Rapid monitoring of diffusion, DC potential, and blood oxygenation changes during global ischemia: effects of hypoglycemia, hyperglycemia, and TTX. Stroke 1999;30:2212-22

21. Auer RN, Siesjo BK. Hypoglycaemia:brain neurochemistry and neuropathology. Bailleires Clin Endocrinol Metab 1993;7:611-25

22. Kiessling M, Xie Y, Kleihues P. Regionally selective inhibition of cerebral protein synthesis in the rat during hypoglycemia and recovery. $J$ Neurochem 1984;43:1507-14

23. Auer RN. Progress review: hypoglycemic brain damage. Stroke 1986;17: 699-708

24. Moritani T, Smoker WR, Sato Y, et al. Diffusion-weighted imaging of acute excitotoxic brain injury. AJNR Am J Neuroradiol 2005;26:216-28

25. Tallroth G, Ryding E, Agardh CD. Regional cerebral blood flow in normal man during insulin-induced hypoglycemia and in the recovery period following glucose infusion. Metabolism 1992;41:717-21

26. Jarjour IT, Ryan CM, Becker DJ. Regional cerebral blood flow during hypoglycaemia in children with IDDM. Diabetologia 1995;38:1090-95

27. Sontineni SP, Lee JM, Porter J. Hypoglycemia-induced pontine infarction in a diabetic male with basilar artery stenosis: insight into the mechanisms of hypoglycemic stroke. Cerebrovasc Dis 2008;25:281-82

28. Lim CC, Gan R, Chan CL, et al. Severe hypoglycemia associated with an illegal sexual enhancement product adulterated with glibenclamide: MR imaging findings. Radiology 2009;250:193-201. Epub 2008 Nov 18 